\title{
ON LEAST SQUARES ESTIMATION OF FOURIER COEFFICIENTS AND OF THE REGRESSION FUNCTION
}

Abstract. The problem of nonparametric function fitting with the observation model $y_{i}=f\left(x_{i}\right)+\eta_{i}, i=1, \ldots, n$, is considered, where $\eta_{i}$ are independent random variables with zero mean value and finite variance, and $x_{i} \in[a, b] \subset \mathbb{R}^{1}, i=1, \ldots, n$, form a random sample from a distribution with density $\varrho \in L^{1}[a, b]$ and are independent of the errors $\eta_{i}, i=1, \ldots, n$. The asymptotic properties of the estimator $\widehat{f}_{N(n)}(x)=\sum_{k=1}^{N(n)} \widehat{c}_{k} e_{k}(x)$ for $f \in L^{2}[a, b]$ and $\widehat{c}^{N(n)}=\left(\widehat{c}_{1}, \ldots, \widehat{c}_{N(n)}\right)^{T}$ obtained by the least squares method as well as the limits in probability of the estimators $\widehat{c}_{k}, k=1, \ldots, N$, for fixed $N$, are studied in the case when the functions $e_{k}, k=1,2, \ldots$, forming a complete orthonormal system in $L^{2}[a, b]$ are analytic.

1. Introduction. Let $y_{i}, i=1, \ldots, n$, be observations at points $x_{i} \in$ $[a, b] \subset \mathbb{R}^{1}$, according to the model $y_{i}=f\left(x_{i}\right)+\eta_{i}$, where $f:[a, b] \rightarrow \mathbb{R}^{1}$ is an unknown square integrable function $\left(f \in L^{2}[a, b]\right)$ and $\eta_{i}, i=1, \ldots, n$, are independent identically distributed random variables with zero mean value and finite variance $\sigma_{\eta}^{2}>0$. Let furthermore the points $x_{i}, i=1, \ldots, n$, form a random sample from a distribution with density $\varrho\left(\varrho \geq 0, \int_{a}^{b} \varrho(x) d x=1\right)$, independent of the observation errors $\eta_{i}, i=1, \ldots, n$. If the functions $e_{k}$, $k=1,2, \ldots$, constitute a complete orthonormal system in $L^{2}[a, b]$, then $f$ has the representation

$$
f=\sum_{k=1}^{\infty} c_{k} e_{k}, \quad \text { where } c_{k}=\frac{1}{b-a} \int_{a}^{b} f(x) e_{k}(x) d x, k=1,2, \ldots
$$

We assume that $e_{k}, k=1,2, \ldots$, are analytic in $(a, b)$ and continuous in $[a, b]$. Examples of orthonormal systems satisfying these requirements are [6] the

1991 Mathematics Subject Classification: Primary 62G07, 62F12.

Key words and phrases: Fourier series, least squares method, regression, consistent estimator. 
trigonometric functions in $L^{2}[0,2 \pi]$ and Legendre polynomials in $L^{2}[-1,1]$.

As an estimator of the vector of coefficients $c^{N}=\left(c_{1}, \ldots, c_{N}\right)^{T}$, for fixed $N$, we take the vector $\widehat{c}^{N}$ obtained by the least squares method:

$$
\widehat{c}^{N}=\arg \min _{a^{N} \in \mathbb{R}^{N}} \sum_{i=1}^{n}\left(y_{i}-\left\langle a^{N}, e^{N}\left(x_{i}\right)\right\rangle\right)^{2},
$$

where $\widehat{c}^{N}=\left(\widehat{c}_{1}, \ldots, \widehat{c}_{N}\right)^{T}, e^{N}(x)=\left(e_{1}(x), \ldots, e_{N}(x)\right)^{T}$.

To such estimators of the Fourier coefficients $c_{k}, k=1, \ldots, N$, there corresponds an estimator of the regression function $f$ of the form

$$
\widehat{f}_{N}(x)=\sum_{k=1}^{N} \widehat{c}_{k} e_{k}(x),
$$

called a projection type estimator [4].

The vector $\widehat{c}^{N}$ can be obtained as a solution of the normal equations

$$
G_{n} \widehat{c}^{N}=g_{n}
$$

where

$$
G_{n}=\frac{1}{n} \sum_{i=1}^{n} e^{N}\left(x_{i}\right) e^{N}\left(x_{i}\right)^{T}, \quad g_{n}=\frac{1}{n} \sum_{i=1}^{n} y_{i} e^{N}\left(x_{i}\right) .
$$

The asymptotic properties of the least squares estimators of the regression function obtained in the same way as described above but for the fixed point design case were examined in [5]. The problem of choosing the regression order for least squares estimators in the case of equidistant observation points was investigated in [4].

In order to investigate the asymptotic properties of the estimators $\widehat{c}_{k}$, $k=1, \ldots, N$, we introduce the probability space $(\Omega, F, P)$, where

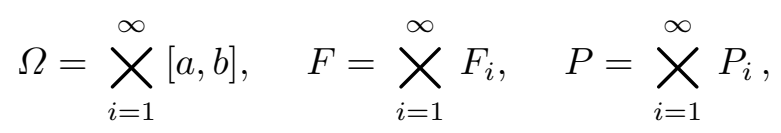

where each $F_{i}, i=1,2, \ldots$, is the $\sigma$-field of Borel subsets of $[a, b]$, and $P$ is a probability measure with the property

$$
P\left(A_{1} \times \ldots \times A_{n} \times \underset{i=n+1}{\times}[a, b]\right)=\left(P_{1} \times \ldots \times P_{n}\right)\left(A_{1} \times \ldots \times A_{n}\right)
$$

for $A_{i} \in F_{i}, i=1, \ldots, n$, with $P_{i}$, for $i=1,2, \ldots$, being the probability measure defined on $F_{i}$ and having density $\varrho$ with respect to the Lebesgue measure $\mu$. The construction and properties of such a probability measure $P$ are described in [2]. The elements of $\Omega$ are denoted by $\omega=\left(x_{1}, x_{2}, \ldots\right)$, $x_{i} \in[a, b], i=1,2, \ldots$

If the distribution of the observation errors $\eta_{i}, i=1,2, \ldots$ (defined on a certain probability space $(\Psi, \Theta, \nu))$, is known, a similar probability space 
can be constructed, with elements of the form $\eta=\left(\eta_{1}, \eta_{2}, \ldots\right)$. From the two above described probability spaces we can of course construct in the usual way the corresponding product space with elements $(\omega, \eta)[2]$.

In the following section we examine the uniqueness of the estimators $\widehat{c}_{k}(\omega, \eta), k=1, \ldots, N$, for fixed $N$, and determine their limits in probability, depending on the density $\varrho$. In the third section we prove that the estimator $\widehat{f}_{N(n)}$ of the regression function corresponding to the Fourier coefficient estimators $\widehat{c}_{k}, k=1, \ldots, N(n)$, is consistent in the sense of the mean square prediction error

$$
D_{N(n)}=\frac{1}{n} E_{\omega} E_{\eta} \sum_{i=1}^{n}\left(f\left(x_{i}\right)-\widehat{f}_{N(n)}\left(x_{i}\right)\right)^{2}
$$

(i.e. $\lim _{n \rightarrow \infty} D_{N(n)}=0$ ), on the condition that the density $\varrho$ is bounded and the sequence $N(n)$ is properly chosen.

\section{Uniqueness and consistency of Fourier coefficient estimators.}

First we check whether the Fourier coefficient estimators $\widehat{c}_{k}, k=1, \ldots, N$, are uniquely determined. In order to do this we need the following two lemmas.

Lemma 2.1. Let $v_{1}, \ldots, v_{n} \in \mathbb{R}^{n}$. The matrix $G_{n}=\sum_{i=1}^{n} v_{i} v_{i}^{T}$ is singular $\left(\operatorname{det} G_{n}=0\right)$ if and only if $v_{1}, \ldots, v_{n}$ are linearly dependent.

Pr o of. Suppose that $G_{n}$ is singular and $v_{1}, \ldots, v_{n}$ are linearly independent. Then there exists a vector $x \neq 0$ for which $G_{n} x=0$ so that

$$
\sum_{i=1}^{n} v_{i}\left(v_{i}^{T} x\right)=\sum_{i=1}^{n}\left\langle v_{i}, x\right\rangle v_{i}=0 .
$$

Since $v_{1}, \ldots, v_{n}$ are linearly independent, $\left\langle v_{i}, x\right\rangle=0$ for $i=1, \ldots, n$. But $\operatorname{span}\left\{v_{1}, \ldots, v_{n}\right\}=\mathbb{R}^{n}$ and consequently $x$ must be zero, contrary to our assumption.

Conversely, if $v_{1}, \ldots, v_{n}$ are linearly dependent, then $\operatorname{dim} \operatorname{span}\left\{v_{1}, \ldots, v_{n}\right\}$ $\left\langle n\right.$ and we can choose $x \neq 0$ such that $\left\langle v_{i}, x\right\rangle=0$ for $i=1, \ldots, n$. Consequently, $G_{n} x=\sum_{i=1}^{n}\left\langle v_{i}, x\right\rangle v_{i}=0$, which means that $G_{n}$ is singular.

By the way, observe that a matrix of the form $G_{m}=\sum_{i=1}^{m} v_{i} v_{i}^{T}$, where $m<n$, is always singular since $\operatorname{dim} \operatorname{span}\left\{v_{1}, \ldots, v_{m}\right\} \leq m$ and there exist nonzero vectors orthogonal to $\operatorname{span}\left\{v_{1}, \ldots, v_{m}\right\}$.

LEMmA 2.2. If $\varrho \in L^{1}[a, b]$ is a density (i.e. $\varrho \geq 0, \int_{a}^{b} \varrho(x) d x=1$ ), then for $n \geq N$ the matrices

$$
G_{n}(\omega)=\frac{1}{n} \sum_{i=1}^{n} e^{N}\left(x_{i}\right) e^{N}\left(x_{i}\right)^{T}, \quad \omega=\left(x_{1}, x_{2}, \ldots\right),
$$


of the normal equations (1) are positive-definite with probability one (in the probability space $(\Omega, F, P))$.

Proof. From the definition of $G_{n}$ it follows that

$$
G_{n+1}(\omega)=\frac{n}{n+1} G_{n}(\omega)+\frac{1}{n+1} e^{N}\left(x_{n+1}\right) e^{N}\left(x_{n+1}\right)^{T} .
$$

So for $x \in \mathbb{R}^{N}$ we have the inequality

$$
\begin{aligned}
& \left\langle G_{n+1}(\omega) x, x\right\rangle \\
& \quad=\frac{n}{n+1}\left\langle G_{n}(\omega) x, x\right\rangle+\frac{1}{n+1}\left\langle e^{N}\left(x_{n+1}\right) e^{N}\left(x_{n+1}\right)^{T} x, x\right\rangle \\
& \quad=\frac{n}{n+1}\left\langle G_{n}(\omega) x, x\right\rangle+\frac{1}{n+1}\left\langle e^{N}\left(x_{n+1}\right), x\right\rangle^{2} \geq \frac{n}{n+1}\left\langle G_{n}(\omega) x, x\right\rangle .
\end{aligned}
$$

Hence $\Omega_{n+1}=\left\{\omega: \operatorname{det} G_{n+1}(\omega)=0\right\} \subset\left\{\omega: \operatorname{det} G_{n}(\omega)=0\right\}=\Omega_{n}$ since the matrices $G_{n}(\omega)$ are nonnegative-definite for $n=1,2, \ldots$ Thus in order to prove that $P\left(\Omega_{n}\right)=0$ for $n \geq N$ it suffices to prove $P\left(\Omega_{N}\right)=0$. (For $n<N$ we have $P\left(\Omega_{n}\right)=1$, which is a simple consequence of our remark after the proof of Lemma 2.1.) By Lemma 2.1,

$$
\operatorname{det} G_{N}(\omega)=0 \Leftrightarrow e^{N}\left(x_{1}\right), \ldots, e^{N}\left(x_{N}\right) \text { are linearly dependent, }
$$

where $\omega=\left(x_{1}, x_{2}, \ldots\right)$, and consequently,

$$
\begin{aligned}
& \Omega_{N}= \\
& \bigcup_{j=1}^{N}\left\{\omega: e^{N}\left(x_{j}\right) \in \operatorname{span}\left\{e^{N}\left(x_{1}\right), \ldots, e^{N}\left(x_{j-1}\right), e^{N}\left(x_{j+1}\right), \ldots, e^{N}\left(x_{N}\right)\right\}\right\} .
\end{aligned}
$$

Moreover,

$$
\begin{aligned}
& P\left(\left\{\omega: e^{N}\left(x_{j}\right) \in \operatorname{span}\left\{e^{N}\left(x_{1}\right), \ldots, e^{N}\left(x_{j-1}\right), e^{N}\left(x_{j+1}\right), \ldots, e^{N}\left(x_{N}\right)\right\}\right\}\right) \\
& \quad=P\left(\left\{\omega: e^{N}\left(x_{N}\right) \in \operatorname{span}\left\{e^{N}\left(x_{1}\right), \ldots, e^{N}\left(x_{N-1}\right)\right\}\right\}\right) \quad \text { for } j=1, \ldots, N,
\end{aligned}
$$

by the properties of the product measure $P_{1} \times \ldots \times P_{N}$. Further,

$$
\begin{aligned}
P\left(\left\{\omega: e^{N}\left(x_{N}\right) \in \operatorname{span}\left\{e^{N}\left(x_{1}\right), \ldots, e^{N}\left(x_{N-1}\right)\right\}\right\}\right) & \\
& =\int_{a}^{b} \ldots \int_{a}^{b} P_{N}\left(A_{N}\right) d P_{1} \ldots d P_{N-1},
\end{aligned}
$$

where $A_{N}=\left(e^{N}\right)^{-1}\left(\operatorname{span}\left\{e^{N}\left(x_{1}\right), \ldots, e^{N}\left(x_{N-1}\right)\right\}\right) \subset[a, b]$, for fixed $x_{1}, x_{2}, \ldots, x_{N-1}$, is the counter-image of the closed linear subspace $\operatorname{span}\left\{e^{N}\left(x_{1}\right), \ldots, e^{N}\left(x_{N-1}\right)\right\}$ by the continuous mapping $[a, b] \ni x_{N} \mapsto$ $e^{N}\left(x_{N}\right) \in \mathbb{R}^{N}$ (the continuity follows from the continuity of $e_{k}, k=1,2, \ldots$ ). Assume now that $P_{N}\left(A_{N}\right)>0$ for fixed $x_{1}, \ldots, x_{N-1}$. This means that the Lebesgue measure $\mu\left(A_{N}\right)$ is positive. For $x_{N} \in A_{N}$ we have

$$
e^{N}\left(x_{N}\right) \in \operatorname{span}\left\{e^{N}\left(x_{1}\right), \ldots, e^{N}\left(x_{N-1}\right)\right\},
$$


and dimspan $\left\{e^{N}\left(x_{1}\right), \ldots, e^{N}\left(x_{N-1}\right)\right\} \leq N-1$. On the other hand,

$$
\operatorname{span}\left\{e^{N}\left(x_{N}\right): x_{N} \in A_{N}\right\}=\mathbb{R}^{N}
$$

since for any $v=\left(v_{1}, \ldots, v_{N}\right)^{T} \in \mathbb{R}^{N}$ orthogonal to the left-hand side

$$
\left\langle e^{N}(x), v\right\rangle=\sum_{k=1}^{N} v_{k} e_{k}(x)=0 \quad \text { for } x \in A_{N},
$$

and the condition $\mu\left(A_{N}\right)>0$ and the analyticity of $e_{k}, k=1,2, \ldots$, imply immediately that $v_{1}=\ldots=v_{N}=0$.

Thus we obtain a contradiction. Consequently, $P_{N}\left(A_{N}\right)=0$ for all $x_{1}, \ldots, x_{N-1}$. This implies that

$$
P\left(\left\{\omega: e^{N}\left(x_{N}\right) \in \operatorname{span}\left\{e^{N}\left(x_{1}\right), \ldots, e^{N}\left(x_{N-1}\right)\right\}\right\}\right)=0
$$

and, by $(2), P\left(\Omega_{N}\right)=0$.

Lemma 2.2 assures that the estimators $\widehat{c}_{1}, \ldots, \widehat{c}_{N}$ obtained from the normal equations (1) are uniquely determined with probability one in the probability space $(\Omega, F, P)$, provided $n \geq N$.

Observe now that the elements of the matrix $G_{n}(\omega)$ in (1) have the form

$$
g_{n i j}(\omega)=\frac{1}{n} \sum_{k=1}^{n} e_{i}\left(x_{k}\right) e_{j}\left(x_{k}\right), \quad \omega=\left(x_{1}, x_{2}, \ldots\right), i, j=1, \ldots, N,
$$

and we easily obtain

$$
E_{\omega} g_{n i j}(\omega)=\frac{1}{n} \sum_{k=1}^{n} E_{\omega} e_{i}\left(x_{k}\right) e_{j}\left(x_{k}\right)=\int_{a}^{b} e_{i}(x) e_{j}(x) \varrho(x) d x=g_{i j} .
$$

The expected value exists because $e_{k}, k=1,2, \ldots$, are continuous in $[a, b]$. Further, since $x_{1}, x_{2}, \ldots$ are chosen independently,

$$
\begin{aligned}
E_{\omega}\left(g_{n i j}(\omega)-g_{i j}\right)^{2} & =\frac{1}{n^{2}} \sum_{k=1}^{n} E_{\omega}\left(e_{i}\left(x_{k}\right) e_{j}\left(x_{k}\right)-g_{i j}\right)^{2} \\
& =\frac{1}{n} \int_{a}^{b}\left(e_{i}(x) e_{j}(x)-g_{i j}\right)^{2} \varrho(x) d x
\end{aligned}
$$

and we see that the elements of $G_{n}(\omega)$ converge in $L^{2}$ to $g_{i j}$ as $n \rightarrow \infty$.

Similarly, for the elements of the right-hand side vector of the normal equations, $g_{n}(\omega, \eta)$, we obtain

$$
\begin{aligned}
E g_{n i}(\omega, \eta) & =\frac{1}{n} \sum_{k=1}^{n} E y_{k} e_{i}\left(x_{k}\right)=\frac{1}{n} \sum_{k=1}^{n} E_{\omega} E_{\eta}\left(f\left(x_{k}\right)+\eta_{k}\right) e_{i}\left(x_{k}\right) \\
& =\frac{1}{n} \sum_{k=1}^{n} E_{\omega} f\left(x_{k}\right) e_{i}\left(x_{k}\right)=\int_{a}^{b} f(x) e_{i}(x) \varrho(x) d x=g_{i}
\end{aligned}
$$


for $i=1, \ldots, N$, because the observation errors $\eta_{k}, k=1,2, \ldots$, have zero mean values; moreover,

$$
\begin{aligned}
E\left(g_{n i}(\omega, \eta)-g_{i}\right)^{2} & =\frac{1}{n^{2}} \sum_{k=1}^{n} E_{\omega}\left(f\left(x_{k}\right) e_{i}\left(x_{k}\right)-g_{i}\right)^{2}+\frac{1}{n^{2}} \sum_{k=1}^{n} E_{\omega} E_{\eta} \eta_{k}^{2} e_{i}^{2}\left(x_{k}\right) \\
& =\frac{1}{n} \int_{a}^{b}\left(f(x) e_{i}(x)-g_{i}\right)^{2} \varrho(x) d x+\frac{1}{n} \sigma_{\eta}^{2} \int_{a}^{b} e_{i}^{2}(x) \varrho(x) d x .
\end{aligned}
$$

This implies that the elements of $g_{n}(\omega, \eta)$ converge in $L^{2}$ to $g_{i}$ as $n \rightarrow \infty$, provided

$$
\int_{a}^{b} f^{2}(x) \varrho(x) d x<\infty .
$$

In that case we can determine the limits in probability of the estimators $\widehat{c}_{1}, \ldots, \widehat{c}_{N}$ by applying the following lemma.

LEMma 2.3. Let $(\Omega, F, P)$ be a probability space. Let $A_{n}(\omega), n=1,2, \ldots$, be a sequence of random matrices of fixed dimension $k$, nonsingular with probability one, and let $y_{n}(\omega)$ be a sequence of random vectors of dimension $k$. If

1) $\lim _{n \rightarrow \infty} A_{n}(\omega) \stackrel{\mathrm{p}}{=} A$ (in probability), where $A$ is a nonsingular matrix,

2) $\lim _{n \rightarrow \infty} y_{n}(\omega) \stackrel{\mathrm{p}}{=} y$,

then the sequence of random vectors $x_{n}(\omega)$ defined with probability one by the equations

$$
A_{n}(\omega) x_{n}(\omega)=y_{n}(\omega), \quad n=1,2, \ldots,
$$

converges in probability to the vector $x$ which is the unique solution of the equation $A x=y$.

Proof. Apply the fact that the elements of the inverse matrix $A^{-1}$ are continuous functions of the elements of the matrix $A$.

In order to use Lemma 2.3 in the case of the normal equations (1) it is enough to show that the matrix $G$ with elements $g_{i j}$ defined in (3) is positive-definite. Clearly, for any $v=\left(v_{1}, \ldots, v_{N}\right)^{T} \in \mathbb{R}^{N}$,

$$
\begin{aligned}
\langle G v, v\rangle & =\sum_{i=1}^{N} \sum_{j=1}^{N} g_{i j} v_{i} v_{j}=\sum_{i=1}^{N} \sum_{j=1}^{N} v_{i} v_{j} \int_{a}^{b} e_{i}(x) e_{j}(x) \varrho(x) d x \\
& =\int_{a}^{b}\left(\sum_{i=1}^{N} v_{i} e_{i}(x)\right)^{2} \varrho(x) d x \geq 0 .
\end{aligned}
$$

Suppose that $\langle G v, v\rangle=0$. Since $\varrho$ is positive on some set with positive Lebesgue measure, $\sum_{i=1}^{N} v_{i} e_{i}(x)=0$ for $x \in \Delta, \mu(\Delta)>0$, and then $v_{1}=$ $\ldots=v_{N}=0$ as already remarked in the proof of Lemma 2.2 . 
We can now formulate the result concerning the convergence in probability of the estimators $\widehat{c}_{1}, \ldots, \widehat{c}_{N}$ for fixed $N$.

THEOREM 2.1. If the density $\varrho \in L^{1}[a, b]$ satisfies $\int_{a}^{b} f^{2}(x) \varrho(x) d x$ $<\infty$, then the estimators $\widehat{c}_{1}, \ldots, \widehat{c}_{N}, N$ being fixed, are for $n \geq N$ uniquely determined with probability one and

$$
\lim _{n \rightarrow \infty} \widehat{c}^{N} \stackrel{\mathrm{p}}{=} G^{-1} g
$$

where $\widehat{c}^{N}=\left(\widehat{c}_{1}, \ldots, \widehat{c}_{N}\right)^{T}, G$ is the matrix with elements

$$
g_{i j}=\int_{a}^{b} e_{i}(x) e_{j}(x) \varrho(x) d x
$$

and $g \in \mathbb{R}^{N}$ is the vector with components

$$
g_{i}=\int_{a}^{b} f(x) e_{i}(x) \varrho(x) d x
$$

$i, j=1, \ldots, N$.

P r o of. The assertion follows from earlier considerations and from Lemmas 2.2 and 2.3 .

The vector $G^{-1} g$ can be characterized more precisely. Namely, consider the functional defined for $z \in \mathbb{R}^{N}$ by the formula

$$
J(z)=\int_{a}^{b}\left(f(x)-\sum_{i=1}^{N} z_{i} e_{i}(x)\right)^{2} \varrho(x) d x, \quad z=\left(z_{1}, \ldots, z_{N}\right)^{T} .
$$

In order to find the points of extrema of $J(z)$ we set its partial derivatives with respect to $z_{i}, i=1, \ldots, N$, to be zero and we obtain the system of linear equations $G z=g$, with $G$ positive-definite. So the components of $\widehat{c}^{N}$ converge in probability to the components of the vector $G^{-1} g$ which minimizes the value of $J(z)$.

In the case of constant density $(\varrho=1 /(b-a))$ we obtain, by $(5)$,

$$
\lim _{n \rightarrow \infty} \widehat{c}^{N} \underline{\underline{\mathrm{p}}} c^{N}, \quad c^{N}=\left(c_{1}, \ldots, c_{N}\right)^{T},
$$

and so $\widehat{c}_{1}, \ldots, \widehat{c}_{N}$ are then consistent estimators of the Fourier coefficients of $f \in L^{2}[a, b]$.

3. Mean square prediction error and choice of the order of regression. Now we deal with the asymptotic properties of the projection type estimator of the regression function $f$ :

$$
\widehat{f}_{N}(x)=\sum_{k=1}^{N} \widehat{c}_{k} e_{k}(x),
$$


where the vector of Fourier coefficient estimators $\widehat{c}^{N}=\left(\widehat{c}_{1}, \ldots, \widehat{c}_{N}\right)^{T}$ is obtained from the normal equations (1),

$$
\widehat{c}^{N}(\omega, \eta)=G_{n}^{-1}(\omega) g_{n}(\omega, \eta)=G_{n}^{-1}(\omega)\left(\frac{1}{n} \sum_{i=1}^{n}\left(f\left(x_{i}\right)+\eta_{i}\right) e^{N}\left(x_{i}\right)\right) .
$$

From the above equality and the decomposition

$$
\begin{aligned}
& f(x)=\sum_{k=1}^{N} c_{k} e_{k}(x)+r_{N}(x)=\left\langle e^{N}(x), c^{N}\right\rangle+r_{N}(x), \\
& \text { where } r_{N}=\sum_{k=N+1}^{\infty} c_{k} e_{k},
\end{aligned}
$$

we obtain

$\widehat{c}^{N}(\omega, \eta)=c^{N}+G_{n}^{-1}(\omega)\left(\frac{1}{n} \sum_{i=1}^{n} r_{N}\left(x_{i}\right) e^{N}\left(x_{i}\right)\right)+G_{n}^{-1}(\omega)\left(\frac{1}{n} \sum_{i=1}^{n} \eta_{i} e^{N}\left(x_{i}\right)\right)$.

Set $a^{N}=(1 / n) \sum_{i=1}^{n} r_{N}\left(x_{i}\right) e^{N}\left(x_{i}\right)$. In view of the equalities

$$
\begin{gathered}
G_{n}=\frac{1}{n} \sum_{i=1}^{n} e^{N}\left(x_{i}\right) e^{N}\left(x_{i}\right)^{T}, \quad E_{\eta}\left(\eta_{i} \eta_{j}\right)=\sigma_{\eta}^{2} \delta_{i j}, \quad i, j=1, \ldots, n, \\
f(x)-\widehat{f}_{N}(x)=\left\langle c^{N}-\widehat{c}^{N}, e^{N}(x)\right\rangle+r_{N}(x)
\end{gathered}
$$

it is easy to show that

$$
\begin{aligned}
E_{\eta}(f(x)- & \left.\widehat{f}_{N}(x)\right)^{2} \\
= & E_{\eta} r_{N}^{2}(x)+2 r_{N}(x) E_{\eta}\left\langle c^{N}-\widehat{c}^{N}, e^{N}(x)\right\rangle+E_{\eta}\left\langle c^{N}-\widehat{c}^{N}, e^{N}(x)\right\rangle^{2} \\
= & r_{N}^{2}(x)-2 r_{N}(x)\left\langle G_{n}^{-1} a^{N}, e^{N}(x)\right\rangle \\
& \quad+\left\langle G_{n}^{-1} a^{N}, e^{N}(x)\right\rangle^{2}+\frac{1}{n} \sigma_{\eta}^{2}\left\langle e^{N}(x), G_{n}^{-1} e^{N}(x)\right\rangle,
\end{aligned}
$$

and further,

$$
\begin{aligned}
\frac{1}{n} \sum_{i=1}^{n} E_{\eta}\left(f\left(x_{i}\right)\right. & \left.-\widehat{f}_{N}\left(x_{i}\right)\right)^{2} \\
& =\frac{1}{n} \sum_{i=1}^{n} r_{N}^{2}\left(x_{i}\right)-2\left\langle G_{n}^{-1} a^{N}, a^{N}\right\rangle+\left\langle G_{n}^{-1} a^{N}, a^{N}\right\rangle+\sigma_{\eta}^{2} \frac{N}{n} .
\end{aligned}
$$

Finally, we obtain the formula

(6) $\frac{1}{n} \sum_{i=1}^{n} E_{\eta}\left(f\left(x_{i}\right)-\widehat{f}_{N}\left(x_{i}\right)\right)^{2}=\frac{1}{n} \sum_{i=1}^{n} r_{N}^{2}\left(x_{i}\right)-\left\langle G_{n}^{-1} a^{N}, a^{N}\right\rangle+\sigma_{\eta}^{2} \frac{N}{n}$. 
Since $G_{n}$ is a.s. positive-definite for $n \geq N$,

$$
0 \leq \frac{1}{n} \sum_{i=1}^{n} E_{\eta}\left(f\left(x_{i}\right)-\widehat{f}_{N}\left(x_{i}\right)\right)^{2} \leq \frac{1}{n} \sum_{i=1}^{n} r_{N}^{2}\left(x_{i}\right)+\sigma_{\eta}^{2} \frac{N}{n} .
$$

In the case of constant density $\varrho=1 /(b-a)$, this inequality yields

$$
\begin{aligned}
E \frac{1}{n} \sum_{i=1}^{n}\left(f\left(x_{i}\right)-\widehat{f}_{N}\left(x_{i}\right)\right)^{2} & \leq \frac{1}{n} \sum_{i=1}^{n} E_{\omega} r_{N}^{2}\left(x_{i}\right)+\sigma_{\eta}^{2} \frac{N}{n} \\
& =\frac{1}{b-a} \int_{a}^{b} r_{N}^{2}(x) d x+\sigma_{\eta}^{2} \frac{N}{n},
\end{aligned}
$$

and since

$$
\frac{1}{b-a} \int_{a}^{b} r_{N}^{2}(x) d x=\frac{1}{b-a} \sum_{k=N+1}^{\infty} c_{k}^{2}
$$

we can rewrite the last inequality in the form

$$
\begin{aligned}
& D_{N}=E \frac{1}{n} \sum_{i=1}^{n}\left(f\left(x_{i}\right)-\widehat{f}_{N}\left(x_{i}\right)\right)^{2} \leq \frac{p_{N}}{b-a}+\sigma_{\eta}^{2} \frac{N}{n}, \\
& \text { where } p_{N}=\sum_{k=N+1}^{\infty} c_{k}^{2} .
\end{aligned}
$$

Since the series $\sum_{k=1}^{\infty} c_{k}^{2}$ is convergent $\left(f \in L^{2}[a, b]\right)$ we conclude from the above inequality that in the case $\varrho=1 /(b-a)$ we have $\lim _{n \rightarrow \infty} D_{N(n)}=0$ provided $\lim _{n \rightarrow \infty} N(n)=\infty$ and $\lim _{n \rightarrow \infty} N(n) / n=0$. The estimator $\widehat{f}_{N(n)}$ is then consistent in the sense of the mean square prediction error $D_{N(n)}$. A similar result holds for the case of bounded density $\varrho$ as one can see from inequality (7).

If we define the prediction error by

$$
d_{N(n)}=\frac{1}{n} \sum_{i=1}^{n}\left(f\left(x_{i}\right)-\widehat{f}_{N(n)}\left(x_{i}\right)\right)^{2},
$$

then the condition $\lim _{n \rightarrow \infty} D_{N(n)}=\lim _{n \rightarrow \infty} E d_{N(n)}=0$ implies of course $\lim _{n \rightarrow \infty} d_{N(n)} \stackrel{\mathrm{p}}{=} 0$. Consequently, the previously proved facts concerning the convergence of the mean square prediction error $D_{N(n)}$ allow us to formulate the following theorem.

Theorem 3.1. If the density $\varrho \in L^{1}[a, b]$ is bounded and the sequence of natural numbers $N(n), n=1,2, \ldots$, satisfies

$$
\lim _{n \rightarrow \infty} N(n)=\infty, \quad \lim _{n \rightarrow \infty} \frac{N(n)}{n}=0,
$$


then the estimator of the regression function

$$
\widehat{f}_{N(n)}=\sum_{k=1}^{N(n)} \widehat{c}_{k} e_{k}
$$

is consistent in the sense of the prediction error $d_{N(n)}\left(\right.$ i.e. $\lim _{n \rightarrow \infty} d_{N(n)} \stackrel{\mathrm{p}}{=}$ 0 in $(\Omega, F, P))$.

Proof. The assertion follows from Lemma 2.2 and from earlier considerations of Section 3 .

Now we consider the problem of choosing the regression order $N$. If we know the values of $p_{N}, N=1,2, \ldots$, and of $\sigma_{\eta}^{2}$, we can choose $N$ according to the criterion

$$
N^{*}=\arg \min _{1 \leq N \leq n}\left(\frac{p_{N}}{b-a}+\sigma_{\eta}^{2} \frac{N}{n}\right)
$$

Then

$$
D_{N^{*}} \leq \frac{p_{N^{*}}}{b-a}+\sigma_{\eta}^{2} \frac{N^{*}}{n}=\min _{1 \leq N \leq n}\left(\frac{p_{N}}{b-a}+\sigma_{\eta}^{2} \frac{N}{n}\right) .
$$

If we only know some estimates $p_{N}^{\prime} \geq p_{N}$ we can replace $p_{N}$ by $p_{N}^{\prime}$ in (8). If the sequence $\left|c_{k}\right|, k=1,2, \ldots$, is decreasing, then $p_{N}$ is a convex function (of $N$ ) and so is $A_{N}=p_{N} /(b-a)+\sigma_{\eta}^{2} N / n$, which cannot then have local minima; we thus have $N^{*}=\max \left\{N: c_{N}^{2} \geq(b-a) \sigma_{\eta}^{2} / n\right\}$ [4].

The values of $p_{N}, N=1,2, \ldots$, can of course be unknown, but we can define the statistic

$$
s_{N}=\frac{1}{n} \sum_{i=1}^{n}\left(y_{i}-\widehat{f}_{N}\left(x_{i}\right)\right)^{2}
$$

for which

$$
\begin{aligned}
E_{\eta} s_{N}= & \frac{1}{n} \sum_{i=1}^{n} E_{\eta}\left(f\left(x_{i}\right)-\widehat{f}_{N}\left(x_{i}\right)+\eta_{i}\right)^{2} \\
= & \frac{1}{n} \sum_{i=1}^{n} E_{\eta}\left(f\left(x_{i}\right)-\widehat{f}_{N}\left(x_{i}\right)\right)^{2}-\frac{2}{n} \sum_{i=1}^{n} E_{\eta} \widehat{f}_{N}\left(x_{i}\right) \eta_{i}+\sigma_{\eta}^{2} \\
= & \frac{1}{n} \sum_{i=1}^{n} E_{\eta}\left(f\left(x_{i}\right)-\widehat{f}_{N}\left(x_{i}\right)\right)^{2}-\frac{2}{n} \sum_{i=1}^{n} E_{\eta}\left\langle\widehat{c}^{N}, e^{N}\left(x_{i}\right)\right\rangle \eta_{i}+\sigma_{\eta}^{2} \\
= & \frac{1}{n} \sum_{i=1}^{n} E_{\eta}\left(f\left(x_{i}\right)-\widehat{f}_{N}\left(x_{i}\right)\right)^{2} \\
& -\frac{2}{n} \sum_{i=1}^{n} E_{\eta}\left\langle G_{n}^{-1}\left(\frac{1}{n} \sum_{j=1}^{n} y_{j} e^{N}\left(x_{j}\right)\right), e^{N}\left(x_{i}\right)\right\rangle \eta_{i}+\sigma_{\eta}^{2}
\end{aligned}
$$




$$
\begin{aligned}
= & \frac{1}{n} \sum_{i=1}^{n} E_{\eta}\left(f\left(x_{i}\right)-\widehat{f}_{N}\left(x_{i}\right)\right)^{2} \\
& -\frac{2}{n} \sum_{i=1}^{n} E_{\eta}\left\langle G_{n}^{-1}\left(\frac{1}{n} \sum_{j=1}^{n} \eta_{j} e^{N}\left(x_{j}\right)\right), e^{N}\left(x_{i}\right)\right\rangle \eta_{i}+\sigma_{\eta}^{2} \\
= & \frac{1}{n} \sum_{i=1}^{n} E_{\eta}\left(f\left(x_{i}\right)-\widehat{f}_{N}\left(x_{i}\right)\right)^{2}-\frac{2}{n^{2}} \sigma_{\eta}^{2} \sum_{i=1}^{n}\left\langle G_{n}^{-1} e^{N}\left(x_{i}\right), e^{N}\left(x_{i}\right)\right\rangle+\sigma_{\eta}^{2} \\
= & \frac{1}{n} \sum_{i=1}^{n} E_{\eta}\left(f\left(x_{i}\right)-\widehat{f}_{N}\left(x_{i}\right)\right)^{2}-2 \sigma_{\eta}^{2} \frac{N}{n}+\sigma_{\eta}^{2} .
\end{aligned}
$$

Hence, remembering the definition of $D_{N}$, we obtain

$$
E s_{N}=E_{\omega} E_{\eta} s_{N}=D_{N}-2 \sigma_{\eta}^{2} \frac{N}{n}+\sigma_{\eta}^{2},
$$

which can be rewritten in the form

$$
E\left(s_{N}+2 \sigma_{\eta}^{2} \frac{N}{n}\right)=D_{N}+\sigma_{\eta}^{2} .
$$

So if we choose $N$ (the order of regression) according to the criterion

$$
N^{*}=\arg \min _{1 \leq N \leq n}\left(s_{N}+2 \sigma_{\eta}^{2} \frac{N}{n}\right)
$$

we can assert that in the mean we obtain those values of $N$ which minimize $D_{N}$ [4]. This kind of criterion for the choice of $N$ is known in the literature as the Mallows-Akaike criterion [1], [3].

4. Conclusions. It is worth remarking that we can obtain a better lower bound for the mean square prediction error than the obvious one $D_{N} \geq 0$. We apply the following lemma proved in [5].

LEMmA 4.1. Let $h=\left(h_{1}, \ldots, h_{n}\right)^{T} \in \mathbb{R}^{n}$. Then

$$
\frac{1}{n^{2}} \sum_{i=1}^{n} \sum_{j=1}^{n} h_{i} h_{j} e^{N}\left(x_{i}\right)^{T} G_{n}^{-1} e^{N}\left(x_{j}\right) \leq \frac{1}{n} \sum_{i=1}^{n} h_{i}^{2} .
$$

Since $a^{N}=(1 / n) \sum_{i=1}^{n} r_{N}\left(x_{i}\right) e^{N}\left(x_{i}\right)$ and $G_{n}>0$ a.s. for $n \geq N$, putting $h_{i}=r_{N}\left(x_{i}\right), i=1, \ldots, n$, by Lemma 4.1 we obtain

$$
0 \leq\left\langle G_{n}^{-1} a^{N}, a^{N}\right\rangle \leq \frac{1}{n} \sum_{i=1}^{n} r_{N}\left(x_{i}\right)^{2}
$$

almost surely for $n \geq N$. Now, taking into account (6) we easily obtain the 
lower and upper bounds for $D_{N}$, valid for $n \geq N$ :

$$
\sigma_{\eta}^{2} \frac{N}{n} \leq D_{N} \leq M_{\varrho} p_{N}+\sigma_{\eta}^{2} \frac{N}{n}, \quad \text { where } M_{\varrho}=\sup _{a \leq x \leq b} \varrho(x) .
$$

From (9) and (10) it follows immediately that in the case when $\varrho$ is bounded and the conditions $\lim _{n \rightarrow \infty} N(n)=\infty$ and $\lim _{n \rightarrow \infty} N(n) / n=0$ are satisfied, $s_{N(n)}$ is an asymptotically unbiased estimator of $\sigma_{\eta}^{2}$.

The lower and upper bounds for $D_{N(n)}$ also allow us to estimate the bias of $s_{N(n)}$ for $n \geq N(n)$, namely

$$
-\sigma_{\eta}^{2} \frac{N(n)}{n} \leq E s_{N(n)}-\sigma_{\eta}^{2} \leq M_{\varrho} p_{N(n)}-\sigma_{\eta}^{2} \frac{N(n)}{n} .
$$

The results presented in the two preceding sections can be easily proved in the case of regression functions $f \in L^{2}(A), A \subset \mathbb{R}^{m}, m>1, \mu(A)<\infty$, and certain complete orthonormal systems of functions (like the functions

$$
\exp (i k x+i l y) / 2 \pi, \quad 0 \leq x, y \leq 2 \pi, k, l=0, \pm 1, \pm 2, \ldots,
$$

forming a complete orthonormal system in $\left.L^{2}([0,2 \pi] \times[0,2 \pi])\right)$.

\section{References}

[1] H. Akaike, A new look at the statistical model identification, IEEE Trans. Automat. Control AC-19 (1974), 716-723.

[2] Y. S. Chow and H. Teicher, Probability Theory, Independence, Interchangeability, Martingales, Springer, Heidelberg, 1978.

[3] C. L. Mallows, Some comments on $C_{p}$, Technometrics 15 (1973), 661-675.

[4] B. T. Polyak and A. B. Tsybakov, Asymptotic optimality of the $C_{p}$ criterion in projection type estimation of a regression function, Teor. Veroyatnost. i Primenen. 35 (1990), 305-317 (in Russian).

[5] E. Rafajłowicz, Nonparametric least-squares estimation of a regression function, Statistics 19 (1988), 349-358.

[6] G. Sansone, Orthogonal Functions, Interscience, New York, 1959.

WALDEMAR POPIŃSKI

RESEARCH AND DEVELOPMENT CENTER OF STATISTICS

AL. NIEPODLEGŁOŚCI 208

00-925 WARSZAWA, POLAND 